\title{
Sleep disordered breathing and chronic obstructive pulmonary disease: a narrative review on classification, pathophysiology and clinical outcomes
}

\author{
Rebecca F. D'Cruz ${ }^{1,2,3}$, Patrick B. Murphy ${ }^{1,2,3}$, Georgios Kaltsakas ${ }^{1,2,3}$ \\ ${ }^{1}$ Lane Fox Respiratory Unit, Guy's and St. Thomas' NHS Foundation Trust, London, UK; ${ }^{2}$ Lane Fox Clinical Respiratory Physiology Research \\ Centre, Guy's and St Thomas' NHS Foundation Trust, UK; ${ }^{3}$ Centre for Human \& Applied Physiological Sciences, King's College London, UK \\ Contributions: (I) Conception and design: All authors; (II) Administrative support: All authors; (III) Provision of study materials or patients: All \\ authors; (IV) Collection and assembly of data: All authors; (V) Data analysis and interpretation: All authors; (VI) Manuscript writing: All authors; (VII) \\ Final approval of manuscript: All authors. \\ Correspondence to: Georgios Kaltsakas. Lane Fox Respiratory Unit, St Thomas' Hospital, London SE1 7EH, UK. \\ Email: georgios.kaltsakas@gstt.nhs.uk.
}

\begin{abstract}
Chronic obstructive pulmonary disease (COPD) causes load-capacity-drive imbalance in both wakefulness and sleep, principally driven by expiratory flow limitation and hyperinflation. Sleep imposes additional burdens to the respiratory muscle pump, driven by changes in respiratory muscle tone, neural respiratory drive and consequences of the supine position. COPD patients are therefore at higher risk of decompensation during sleep, which may manifest as altered sleep architecture, isolated nocturnal desaturation, sleep hypoventilation and restless legs. Each form of sleep disordered breathing in COPD is associated with adverse clinical and patient-reported outcomes, including increased risk of exacerbations, hospitalisation, cardiovascular events, reduced survival and poorer quality of life. COPD-obstructive sleep apnoea (OSA) overlap syndrome represents a distinct clinical diagnosis, in which clinical outcomes are significantly worse than in either disease alone, including increased mortality, risk of cardiovascular events, hospitalisation and exacerbation frequency. Sleep disordered breathing is under-recognised by COPD patients and their clinicians, however early diagnosis and management is crucial to reduce the risk of adverse clinical outcomes. In this narrative review, we describe the pathophysiology of COPD and physiological changes that occur during sleep, manifestations and diagnosis of sleep disordered breathing in COPD and associated clinical outcomes.
\end{abstract}

Keywords: Chronic obstructive pulmonary disease (COPD); sleep; sleep disordered breathing; COPDobstructive sleep apnoea (OSA) overlap; respiratory physiology; pulmonary mechanics

Submitted Jun 09, 2020. Accepted for publication Aug 17, 2020.

doi: 10.21037/jtd-cus-2020-006

View this article at: http://dx.doi.org/10.21037/jtd-cus-2020-006

\section{Introduction}

Optimal sleep duration and quality is fundamental to the maintenance of physical and psychological wellbeing but is associated with physiological changes that impose burdens onto the respiratory muscle pump. Such burdens are of limited significance in healthy subjects however may be amplified in respiratory diseases in which there is load-capacity-drive imbalance during wakefulness.
Chronic obstructive pulmonary disease (COPD) is one of the most prevalent respiratory diseases worldwide. Pathological inflammatory responses to inhaled noxious particles lead to alveolar destruction, mucus hypersecretion and hyperinflation which impose elastic, resistive and threshold loads onto the respiratory system. In addition to the characteristic symptoms of breathlessness, cough and sputum production, COPD patients frequently report poor 
sleep and nocturnal respiratory symptoms. There appears to be a bidirectional relationship between sleep quality and clinical outcomes in this population, with sleep disturbance promoting systemic inflammation, impaired immune function, physical inactivity and altered cognition, which potentially impacts on medication adherence, leading to adverse clinical outcomes, and nocturnal COPD symptoms, reduced physical activity, culprit medications and anxiety and depression are all associated with altered sleep architecture (Figure 1). In this review, we provide overviews of COPD and normal sleep physiology, delineate the classification of sleep disordered breathing in COPD and review the impact of sleep disordered breathing on clinical outcomes in COPD.

We present the following article/case in accordance with the Narrative review checklist (available at http://dx.doi. org/10.21037/jtd-cus-2020-006).

\section{Pathophysiology of COPD}

COPD is estimated to affect around 400 million people worldwide and has a prevalence of approximately $10 \%$, although many millions are thought to be undiagnosed (1). The burden of COPD is projected to rise, with deaths attributable to the disease rising from 3 to 7 million between 2016 and 2060 (2). COPD develops as a consequence of inhalation of noxious particles, typically cigarette smoking, in suspectable individuals. Exposure to such stimuli lead to accumulation of inflammatory cells in the airways, predominantly CD8 T-lymphocytes, macrophages and neutrophils. These cells promote fibroblast activity, leading to abnormal airway remodelling and increased airway resistance, and protease-antiprotease imbalance, causing elastin breakdown and reduced elastic recoil of lung parenchyma. Oxidants released from airway inflammatory cells and directly from cigarette smoke also promote mucus hypersecretion, which is an additional resistive load (3). This abnormal inflammatory response ultimately causes expiratory flow limitation, which is the pathophysiological hallmark of COPD. Expiratory flow limitation results in gas trapping, or hyperinflation (4). This imposes elastic and threshold loads and causes respiratory muscle shortening and abnormal chest wall geometry, thus impairing the force generating capacity of the respiratory muscle pump and reducing the efficiency with which inspiratory muscle contraction in translated to inspiratory airflow (5). This leads to increased neural respiratory drive to maintain ventilation, which is perceived as breathlessness (Figure 2). Exacerbations of COPD are acute deteriorations in breathlessness, cough and/or sputum production that result in additional therapy (6). During exacerbations, there is an acute rise in airway resistance due to increased airway inflammation, smooth muscle constriction and excessive production of tenacious sputum, which further increases the end-expiratory volume. COPD exacerbations are associated with poor clinical outcomes, including accelerated lung function decline, reduced health-related quality of life and increased risk of hospitalisation and death (6).

\section{Normal sleep}

Sleep may be categorised into rapid eye movement (REM) and non-REM stages. Sleep architecture in healthy subjects typically progresses through increasingly deep stages of non-REM sleep, with stages 1-2 considered light and $\mathrm{N} 3$ as deep, interrupted by periods of REM sleep. The

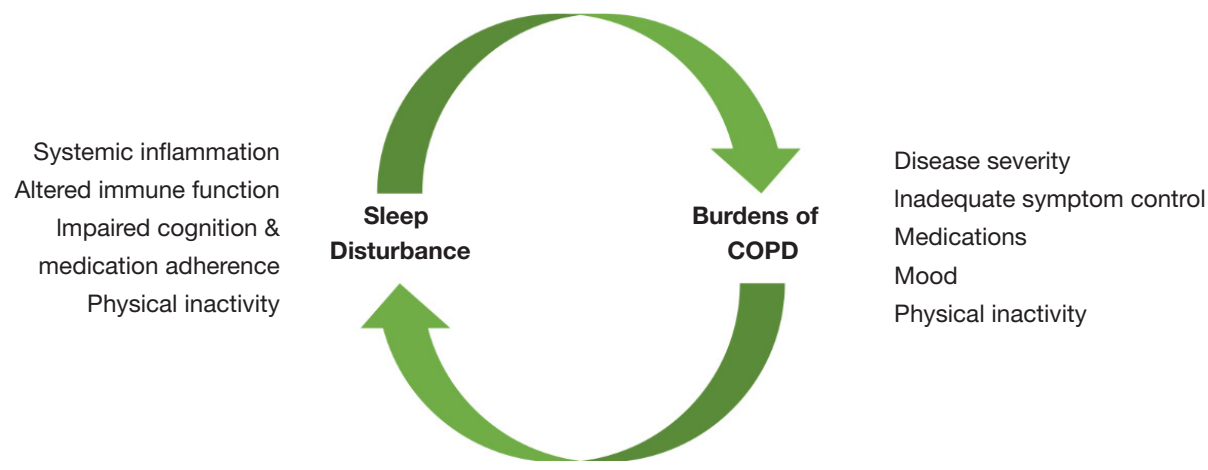

Figure 1 Illustration of the bidirectional relationship between sequelae of sleep-disturbance and adverse clinical outcomes in chronic obstructive pulmonary disease (COPD). 


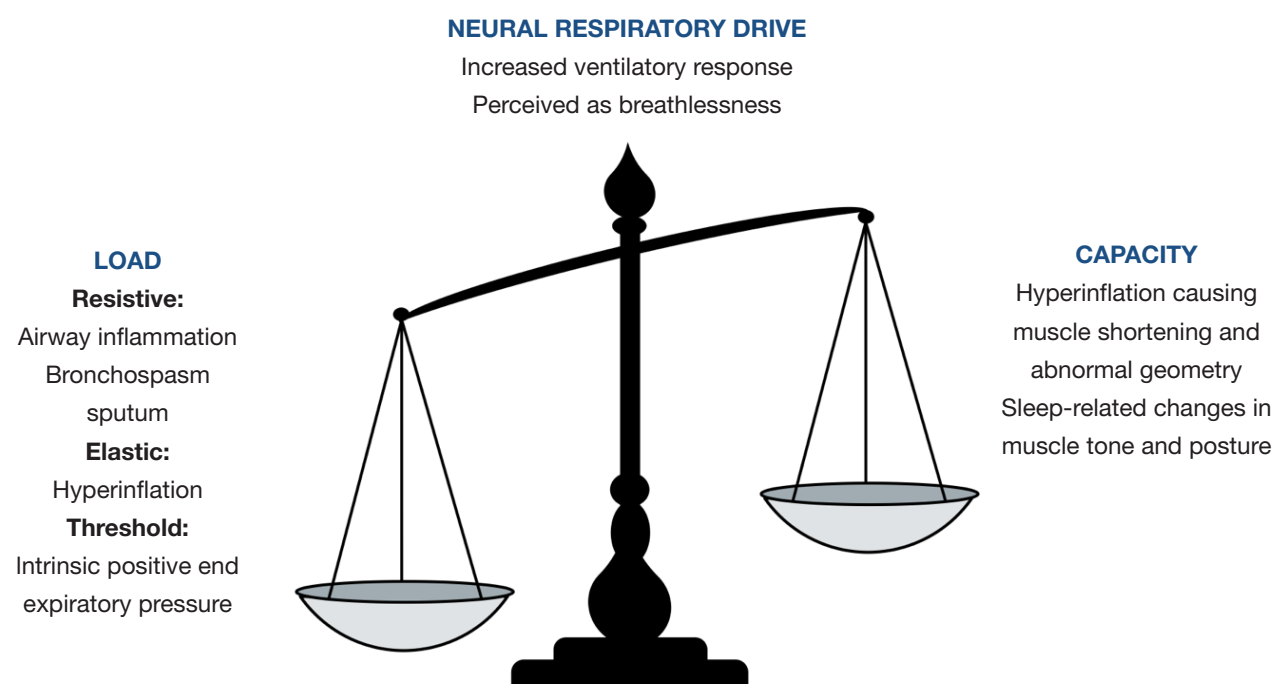

Figure 2 Schematic representation of load-capacity-drive imbalance of the respiratory muscle pump during exacerbations of chronic obstructive pulmonary disease (COPD).

normal physiology of sleep imposes burdens onto the loadcapacity-drive relationship of the respiratory muscle pump. Generalised postural muscle hypotonia occurs throughout sleep and is most profound during REM. During this stage, there is almost complete loss of tone of intercostal muscles with relative preservation of diaphragm activity and thus increased reliance on the diaphragm to maintain ventilation $(7,8)$. A rapid shallow breathing pattern is adopted in sleep which, in combination with reduced inspiratory muscle activity, results in reduced tidal volumes particularly during REM compared to wakefulness (9). Reduced tidal volumes may be accompanied by compensatory increases in respiratory rate during sleep, causing minute ventilation to remain unchanged or only slightly reduced compared to wakefulness $(8,10)$. In healthy subjects with habitual snoring, upper airway resistance is increased throughout all stages of sleep. This is associated with increased end-tidal carbon dioxide $\left(\mathrm{CO}_{2}\right)$, which falls with externally applied positive airway pressure suggesting that this resistive load is an important contributor to sleep-related hypoventilation (11). The supine position adopted during sleep cranially displaces the diaphragm, which imposes additional load and reduces the capacity of respiratory muscles. This leads to reduced end-expiratory lung volume and reduced functional residual capacity (FRC) (12). Normal sleep is also associated with blunted ventilatory responses to both hypoxaemia and hypercapnia and significant reductions in oxygen saturation and increased arterial partial pressure of $\mathrm{CO}_{2}\left(\mathrm{PaCO}_{2}\right)$ of up to $6 \mathrm{mmHg}(0.8 \mathrm{kPa})$ may occur in healthy subjects $(13,14)$.

\section{Sleep disordered breathing and COPD}

The physiological burdens imposed onto the respiratory system during sleep are of little consequence in healthy subjects but are magnified in patients with load-capacitydrive imbalance during wakefulness, such as in COPD. These patients are at risk of decompensating during sleep which can largely be attributed to (I) sleep-related inspiratory muscle tone and (II) the supine position.

\section{Inspiratory muscle tone}

Sleep-related inspiratory muscle hypotonia leads to reliance on the diaphragm to maintain ventilation. In COPD, the diaphragm is mechanically inefficient due to hyperinflation. Measurement of diaphragm electromyography with multipair oesophageal electrode catheters demonstrates that COPD patients have significantly higher diaphragm activity during wakefulness than healthy subjects to achieve comparable rates of minute ventilation. During sleep, in contrast to healthy subjects, diaphragm activity in COPD falls precipitously, to $31 \%$ and $49 \%$ of awake values during NREM and REM sleep, respectively (Figure 3). This is accompanied by large falls in minute ventilation, principally mediated by reduced tidal volumes (8). Furthermore, accessory muscle activity, present during wakefulness and 
NREM sleep in COPD, falls precipitously and may be entirely absent during REM (7). This, in combination with normal REM-related intercostal muscle hypotonia, leads to greater reliance on the functionally impaired diaphragm to maintain ventilation.

\section{Supine posture}

The supine posture is mechanically disadvantageous to pulmonary mechanics in healthy subjects and has additional implications in COPD. Increased airway resistance from smooth muscle hypertrophy, mucus hypersecretion, and alveolar damage leading to destruction of the alveolar attachments to small airways results in increased closing capacity (CC), meaning that airways collapse earlier, at higher lung volumes. The supine position decreases FRC. Once FRC-CC is less than zero, small airway closure occurs during tidal breathing, therefore their attached alveoli do not participate fully in gas exchange. This leads to or exacerbates pre-existing ventilation-perfusion mismatch, which causes hypoxaemia. Inspiratory muscle strength is also affected by posture, with falls in maximal inspiratory pressure observed in COPD patients in the supine position compared to the seated position (15). It is thus unsurprising that COPD patients preferentially adopt a lateral position during sleep, with only $10 \%$ preferring to sleep supine (16).

Physiological and postural changes that occur during sleep thus expose COPD patients to greater risk of developing sleep disordered breathing. This may manifest as (I) altered sleep architecture, (II) isolated nocturnal desaturation, (III) sleep hypoventilation and (IV) restless legs. COPD may co-exist with obstructive sleep apnoea (OSA), termed "overlap syndrome", which represents a distinct clinical entity and will be reviewed separately. A summary of sleep disordered breathing in COPD is provided in Table 1.

\section{Altered sleep architecture}

Sleep fragmentation is common in COPD. Up to $78 \%$ of patients report some form of nocturnal sleep disturbance and the prevalence rises with increasing severity of airflow limitation (24). Polysomnographic evaluation of COPD patients reveals prolonged sleep latency, frequent arousals (occurring on average 15 times per hour), reduced sleep efficiency, total sleep time and N3 and REM sleep and increased wakefulness after sleep onset compared to age, gender, BMI and smoking status matched controls (Table 1) (25-27). These changes in sleep architecture may be partially attributable to COPD-related symptoms, with evening or night-time cough and wheeze reported by up to a quarter of patients and higher levels of sleep disturbance reported in patients who produce frequent sputum compared to non-productive patients $(16,28)$. Anxiety and depression are common co-morbidities in COPD, and patients are more likely to report trouble falling asleep, racing thoughts at bedtime and being unable to sleep for days compared to matched controls, which likely contribute to sleep fragmentation (27). COPD medications may also impact upon sleep. Theophylline improves nocturnal ventilation however is associated with worse polysomnographic indices of sleep quality whilst regular inhaled ipratropium bromide improves nocturnal oxygenation and objective sleep quality $(29,30)$. Sleep analysis of smokers and nonsmokers reveals differences in electroencephalogram indices in early sleep and smokers are more likely to report poor quality sleep, which may be attributed to nicotine withdrawal during sleep (31).

Associations between objectively measured alterations in sleep architecture and clinical outcomes in COPD have not been established. Subjective sleep quality and self-reported disturbance is associated higher risk of exacerbations and emergency healthcare utilisation, more severe daytime breathlessness and cough compared to those without nighttime symptoms $(17,24,32)$.

\section{Isolated nocturnal desaturation}

Nocturnal desaturation occurs in young healthy subjects, in whom saturations under $90 \%$ for up to $10 \%$ of total sleep time may be considered normal, and it is also considered to be part of the normal aging process $(33,34)$. COPD patients are at greater risk of clinically significant nocturnal desaturation, which is considered to be present when more than $30 \%$ of total sleeping time is spent with oxygen saturations under $90 \%$ or mean overnight oxygen is under 90\% (35) (Table 1, Figure 4). Its prevalence amongst non-smoking COPD patients is estimated to be low, at approximately 5\% (36). Daytime oxygenation is an independent predictor of nocturnal desaturation and in patients with mild to moderate daytime hypoxaemia $\left(\mathrm{PaO}_{2}\right.$ 7.4-9.2 $\mathrm{kPa}$ ), this prevalence rises to $49-70 \%(36-38)$. Altered sleep architecture in COPD frequently co-exists with nocturnal oxygen desaturation. However, studies evaluating the effects of nocturnal oxygen therapy on sleep fragmentation are conflicting and beneficial effects on arousals, total sleep time and sleep stage distribution (reduced light sleep and increased REM) may only be 


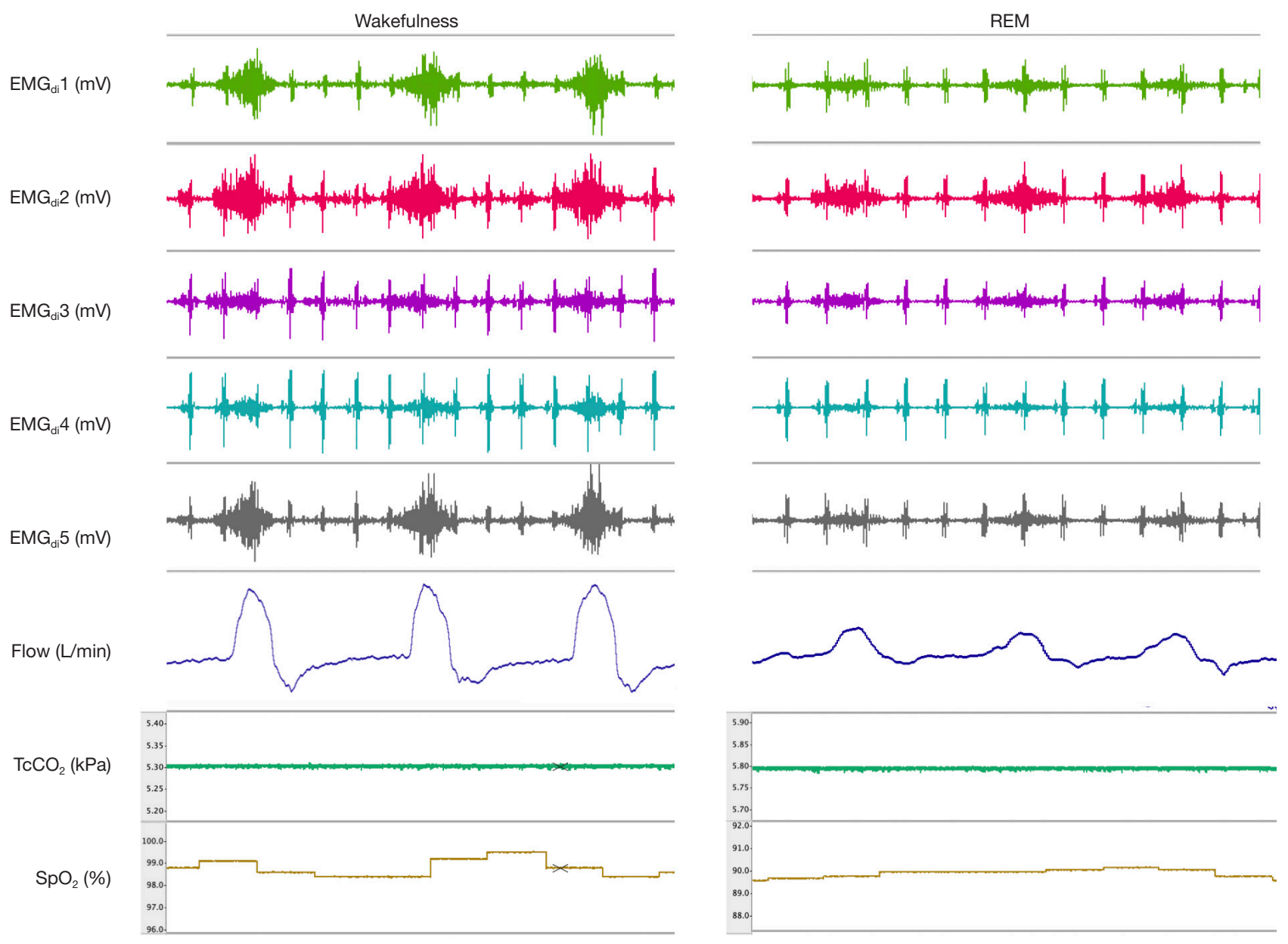

Figure 3 Physiological changes during wakefulness and rapid eye movement (REM) sleep including diaphragm electromyography (EMG $\mathrm{di}_{\mathrm{di}}$ pairs 1-5) from a multipair oesophageal electrode catheter, air flow at the mouth from a pneumotachograph, transcutaneous carbon dioxide $\left(\mathrm{TcCO}_{2}\right)$ and peripheral oxygen saturation $\left(\mathrm{SpO}_{2}\right)$. Data obtained from a patient with chronic obstructive pulmonary disease.

evident amongst patients with more profound daytime hypoxaemia $(25,39)$. Furthermore, whilst nocturnal oxygen administration and regular long-acting bronchodilation improves nocturnal oxygenation without inducing hypercapnia, neither improve subjective sleep quality (40-42).

The clinical significance of isolated nocturnal desaturation in COPD has yet to be established, however patients with mild daytime hypoxaemia and nocturnal desaturation have worse survival than those without nocturnal desaturation (43). Cardiovascular disease commonly co-exists with COPD, with comorbid patients experiencing higher mortality and morbidity, including increased risks of exacerbations, cardiovascular events and hospitalisation, poorer health status and increased symptom burden than patients with either disease alone (44). The pathophysiological mechanisms underlying these poor clinical outcomes is incompletely understood however chronic intermittent hypoxaemia is likely a key component. Healthy subjects exposed to chronic intermittent hypoxaemia $\left(\mathrm{SaO}_{2} 84 \%\right)$ develop raised mean arterial blood pressure due to increased carotid body chemoreceptiveness, leading to increased sympathetic activation and peripheral vascular resistance (45). In COPD, nocturnal hypoxaemia is associated with electrocardiographic changes, with tachycardia, atrial and ventricular ectopics, prolonged $\mathrm{QT}_{c}$ interval, ST depression and right bundle branch block all observed and abolished with oxygen application (46). Chronic intermittent hypoxia may also be associated with increased pulmonary and systemic inflammation. 
Table 1 Classification of sleep disordered breathing in chronic obstructive pulmonary disease (COPD), diagnostic criteria and associated clinical outcomes (data collated from references 7,8,17-23)

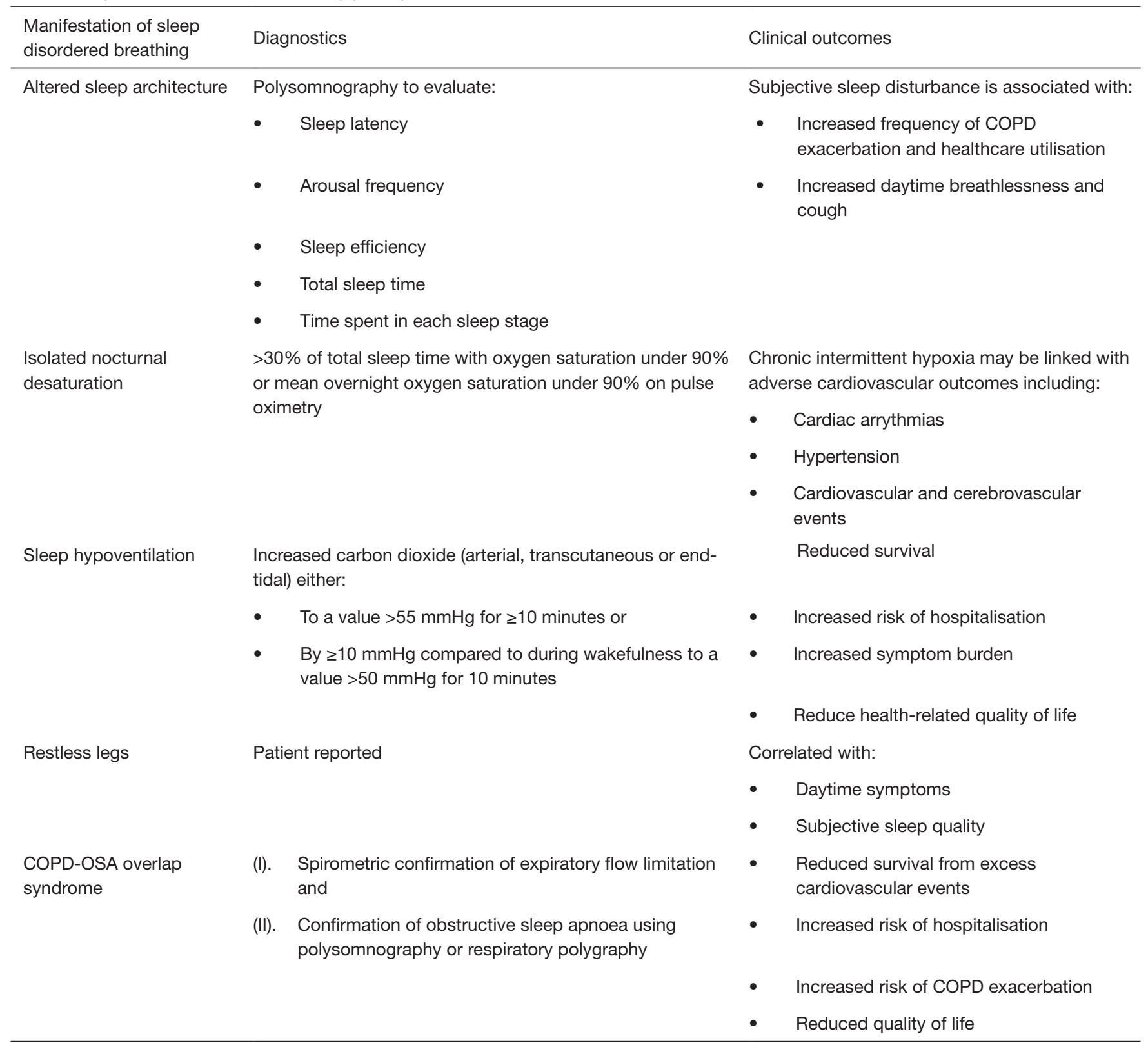

Airway walls of smokers and COPD patients are typically infiltrated with macrophages and T-lymphocytes with the presence of neutrophils in the airway lumen and stable COPD patients with comorbid cardiovascular disease have raised serum levels of fibrinogen, IL-6 and IL-8 than in COPD patients without cardiovascular disease $(47,48)$. These processes likely lead to the development of increased arterial stiffness which is observed in COPD patients and is an independent predictor of cardiovascular events (18). The effects of nocturnal oxygen therapy in COPD patients with isolated nocturnal desaturation (with or without daytime hypoxaemia) on cardiovascular risk is currently unknown. Pulmonary haemodynamics have also been investigated in patients with daytime hypoxaemia and nocturnal desaturation, with no correlation found between pulmonary artery pressure and severity and duration of nocturnal hypoxaemia (38).

Isolated nocturnal desaturation does not appear to have a 


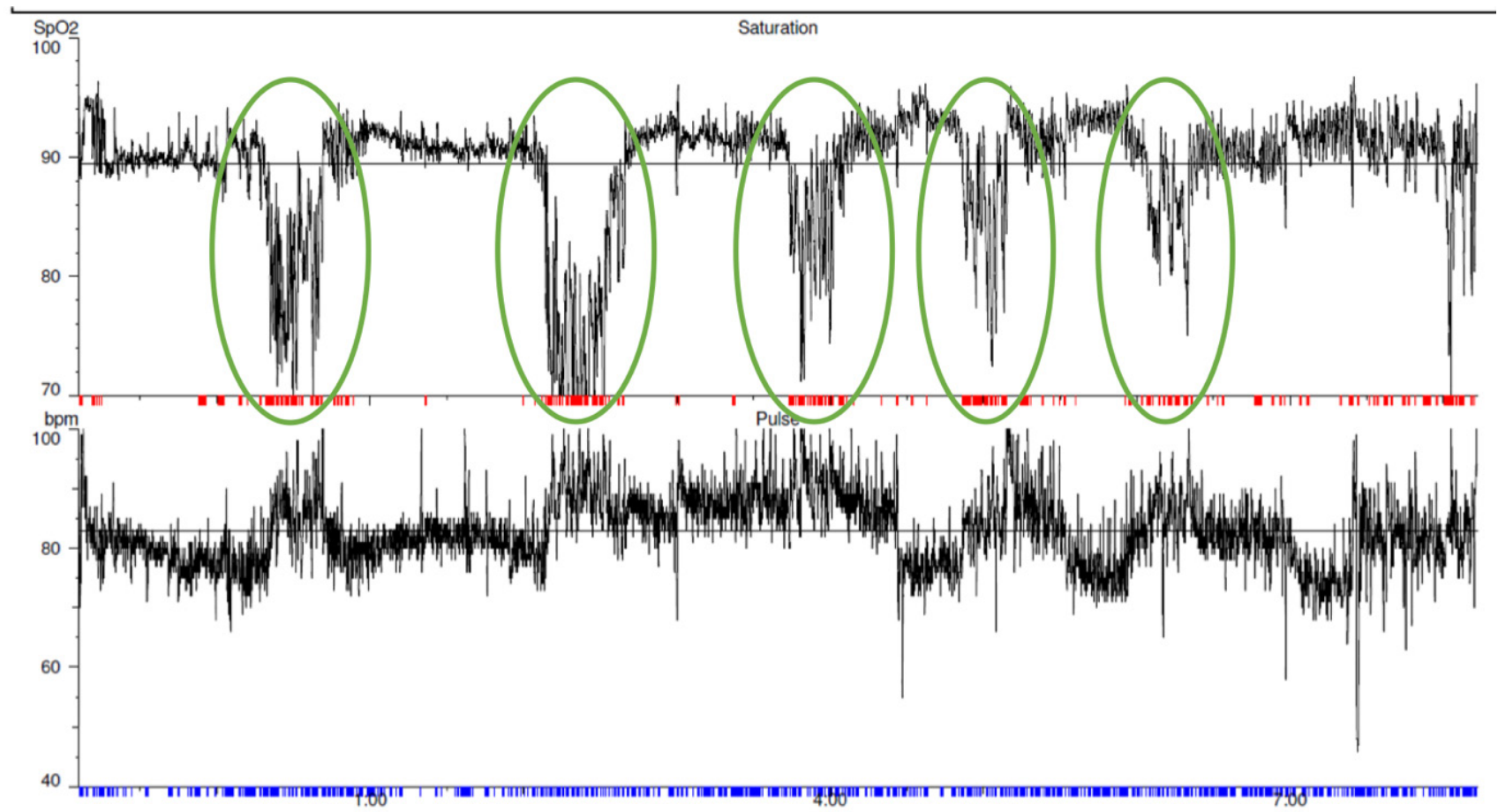

Figure 4 Example of overnight oximetry in a patient with nocturnal hypoxia. Episodes of nocturnal desaturation that are likely related to rapid eye movement (REM) stage sleep are highlighted in green.

detrimental impact on patient-reported outcomes, with no differences in subjective sleep quality, daytime somnolence or health-related quality of life observed between COPD patients with and without nocturnal desaturation (36). However, minimum and mean overnight oxygen saturation may be independent predictors of psychiatric-related sleep disturbance, which is more common in COPD compared to matched controls (27).

\section{Sleep hypoventilation}

Episodes of sleep hypoventilation are defined as increased in carbon dioxide $\mathrm{PaCO}_{2}$, transcutaneous $\left(\mathrm{TcCO}_{2}\right)$ or end-tidal $\left.\left(\mathrm{ETCO}_{2}\right)\right]$ either (I) to a value $>55 \mathrm{mmHg}$ for $\geq 10$ minutes or (II) by $\geq 10 \mathrm{mmHg}$ compared to during wakefulness to a value $>50 \mathrm{mmHg}$ for 10 minutes (Table 1) (49). Sleep hypoventilation is observed in up to $43 \%$ of COPD patients with chronic respiratory failure (daytime $\mathrm{PaCO}_{2}$ $46 \mathrm{mmHg} / 6 \mathrm{kPa}$ ) and is more prevalent with increasing severity of airflow limitation (50). There is a less welldefined cohort of eucapnic COPD patients with isolated nocturnal hypoventilation who may be at increased risk of frequent exacerbations and pulmonary artery hypertension, however the prevalence and clinical relevance of this phenomenon has not been prospectively evaluated $(51,52)$.
Benzodiazepine, opiate and hypnotic medications improve sleep efficiency and are effective in palliating chronic breathlessness. However, they are associated with reduced tidal volumes, minute ventilation and neural respiratory drive during sleep and so should be used judiciously, particularly in hypercapnic patients $(53,54)$.

Sleep hypoventilation in COPD manifests both as transient and sustained increases in $\mathrm{PaCO}_{2}$, with REMrelated rises throughout the night and elevated morning $\mathrm{PaCO}_{2}$ compared with $\mathrm{PaCO}_{2}$ from the preceding evening (50). Nocturnal hypercapnia in COPD reflects reduced minute ventilation, which falls by up to $19 \%$ during non-REM sleep and 36\% during REM compared to wakefulness (55). Sleep-related hypoventilation is likely a consequence of (I) excess load imposed by increased upper airways resistance and impaired dilatory upper airways response to hypercapnia, (II) impaired capacity due to functional diaphragm impairment and (III) blunted neural respiratory drive, as measured with airway occlusion pressure $\left(\mathrm{P}_{0.1}\right)$ and diaphragm electromyography, which occur during all stages of sleep compared to wakefulness, and particularly during REM (8,55-57) (Figure 5). Nocturnal hypercapnia does not impact upon objective sleep quality, with total sleep time, distribution of sleep 


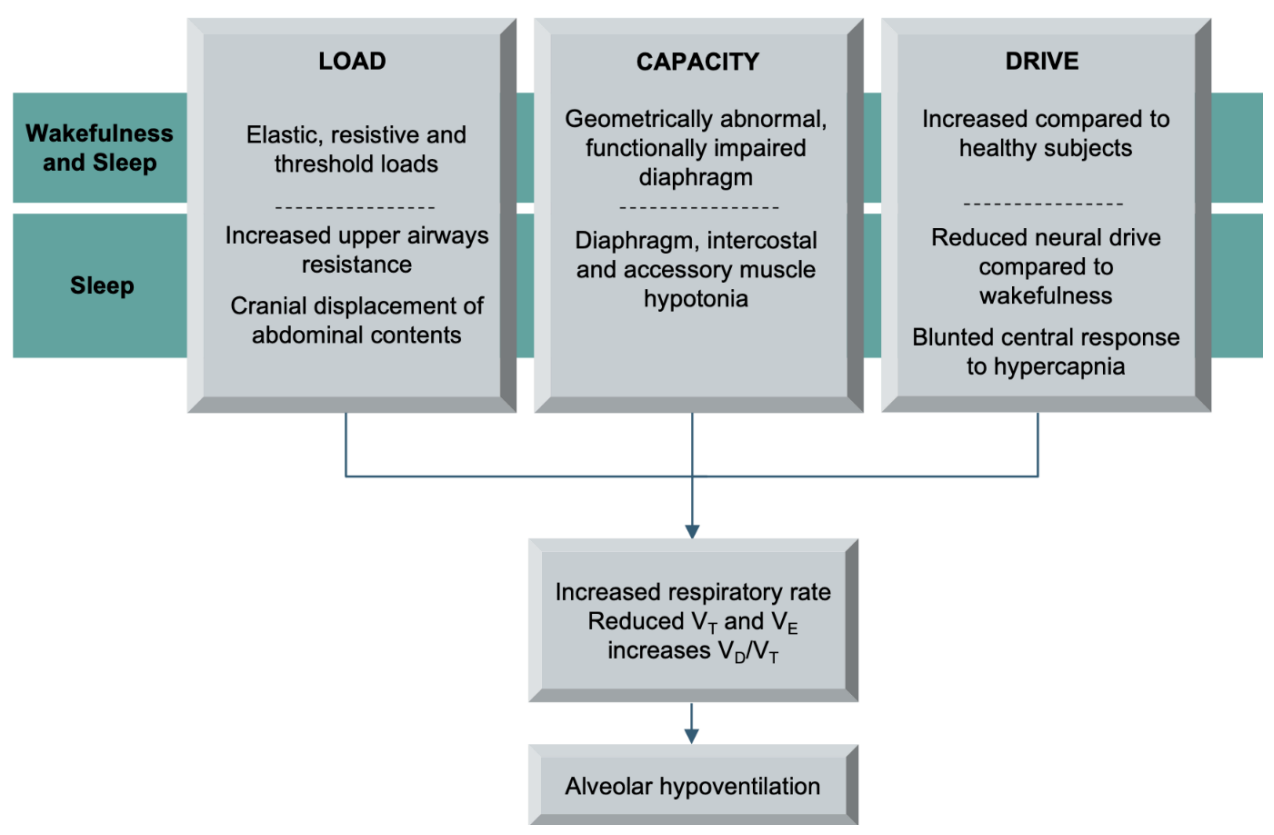

Figure 5 Excess load-capacity-drive imbalance during rapid eye movement (REM)-sleep in COPD leading to nocturnal hypoventilation. Abbreviations: VT, tidal volume; VE, minute ventilation; VD, dead space volume.

stages and arousal, hypopnoea and apnoea frequency comparable between COPD patients with and without daytime hypercapnia (51).

Untreated chronic respiratory failure in COPD is associated with poor clinical outcomes, including higher risk of hospitalisation, increased symptom burden and impaired health related quality of life (6). Median survival is 2.5 years, which is significantly lower than in respiratory failure from other causes $(19,58)$. Nocturnal non-invasive bilevel positive pressure ventilation (NIV) improves physiological and clinical outcomes in COPD in both stable hypercapnic patients and those with persistent hypercapnia following hypercapnic exacerbation requiring acute NIV $(59,60)$. In stable non-obese COPD patients, nocturnal NIV is associated with small increases in duration of REM sleep, reduced nocturnal rises in $\mathrm{CO}_{2}$ and fewer apnoeic/ hypopnoeic events during inpatient setup (61). In the long-term, when targeted to reduce daytime hypercapnia, nocturnal NIV improves survival, functional exercise capacity and health-related quality of life 12 months after initiation (59). Applying a high intensity approach (high inspiratory pressure and high back up rate) does not produce any difference in overnight oxygen saturation, $\mathrm{TcCO}_{2}$, or sleep efficiency (as measured with actigraphy) compared to a high pressure strategy (high inspiratory pressure, low backup rate) and may adversely affect health related quality of life (62). Volume targeted NIV may enable delivery of lower pressures and, when titrated with polysomnography, is associated with improved patient-ventilator asynchrony, subjective comfort, morning breathlessness and nocturnal $\mathrm{TcCO}_{2}(63)$. NIV devices with auto-titrating algorithms deliver a predefined tidal volume by adjusting delivered pressures and backup rate, so-called average or intelligent volume assured pressure support (AVAPS or iVAPS). These appear to improve nocturnal ventilation without adversely affecting subjective and objective indices of sleep quality $(64,65)$. Following exacerbations requiring acute NIV, patients with persistent hypercapnia have improved daytime oxygenation and nocturnal hypoventilation when treated with nocturnal NIV and home oxygen compared to home oxygen alone, in addition to prolonged time to readmission or death, lower all-cause mortality and reduced exacerbation frequency (60). To date, there have been no studies evaluating physiological or clinical outcomes following application of positive airway pressure in isolated nocturnal hypoventilation.

\section{Restless legs syndrome}

Restless legs syndrome is characterised by a subjective compelling urge to move the limbs, typically worse at night 
and relieved by activity (66). It is more common in COPD, affecting approximately one third of patients, with a female preponderance $(20,21)$. Severity of restless legs is correlated with both daytime breathlessness and subjective sleep quality, with patients commonly reporting non-refreshing sleep, daytime somnolence and low mood $(20,21)$.

\section{COPD-obstructive sleep apnoea (OSA) overlap}

OSA causes repetitive upper airway obstruction with transient hypoxaemia during sleep with daytime somnolence. It is more prevalent amongst men and those with hypertension or higher body mass index (BMI), affecting $10-17 \%$ of the middle-aged adult population $(67,68)$. OSA is associated with increased mortality and morbidity from cardiovascular disease, including coronary artery disease, heart failure, arrythmias and stroke. The underlying pathophysiological mechanisms behind these poor clinical outcomes remain unclear, however strong associations have been identified between OSA and endothelial dysfunction, oxidative stress, systemic inflammation, coagulopathy and altered sympathetic drive which improve with overnight continuous positive airway pressure therapy (69). Given the high prevalence of COPD and OSA worldwide, the development of both diseases in an individual (the so-called "overlap syndrome") by chance alone is not unlikely. The prevalence of COPDOSA overlap has been estimated at $1-3.6 \%$ of the general population, and studies evaluating patients with established diagnoses of either COPD or OSA indicate the prevalence of the overlap syndrome is up to $66 \%$ and $56 \%$, respectively $(70,71)$. These estimates must however be interpreted with caution since they are obtained from non-randomised trials (therefore subject to selection bias) with variable diagnostic criteria for each disease (spirometric values, definitions of hypopnoea, apnoea-hypopnoea index (AHI) cut-off values). Furthermore, the common occurrence of isolated nocturnal desaturation even in mild COPD may overestimate OSA prevalence in this cohort (67).

To date, there have been no studies demonstrating causal relationships between pre-existing COPD or OSA and development of the overlap syndrome. It has been speculated that COPD patients are at increased risk of upper airway obstruction during sleep as a consequence of rostral fluid shifts, which may be more significant with right heart failure, and generalised muscle atrophy, which is common in COPD and may predispose to pharyngeal muscle weakness $(72,73)$. Corticosteroids, which are commonly used in COPD, could theoretically increase risk of sleeprelated upper airway obstruction due to the common side effects of myopathy, central obesity and fluid retention (74). During NREM, minute ventilation falls significantly compared to wakefulness in patients with COPD, OSA and those with overlap. During NREM, neural respiratory drive falls in COPD, increases in OSA and remains stable in COPD-OSA overlap and upper airway resistance, which remains stable during sleep in COPD, increases in OSA and COPD-OSA overlap during NREM. Thus in COPD-OSA overlap, sleep hypoventilation is more likely a consequence of increased upper airway resistance than reduced neural drive in these patients (75). Furthermore, worsening airflow limitation and gas trapping in patients with OSA is associated with reduced upper airway dilation responsiveness to increased neural drive and increased instability of ventilatory control (76).

Conversely, data from clinical trials suggest that hyperinflation caused by expiratory airflow limitation may be protective against the development of OSA. Inverse relationships have been reported between AHI and forced expiratory volume in 1 second or radiological emphysema in patients with COPD-OSA overlap $(68,77)$. These findings are consistent with observations that increased end-expiratory lung volume is associated with reduced upper airway collapsibility during NREM sleep $(78,79)$. Whilst the mechanism underlying the negative correlation between airflow limitation and $\mathrm{AHI}$ have not been defined in patients with COPD-OSA overlap, it has been demonstrated that COPD patients effectively adapt their respiratory cycle in response to inspiratory airflow limitation compared to BMI- and AHI-matched smokers without COPD, as demonstrated by prolonged inspiratory duty cycle $\left(\mathrm{T}_{\mathrm{i}} / \mathrm{T}_{\text {tot }}\right)$ with a corresponding reduction in upper airway collapsibility (80). Reduced upper airway collapsibility observed during sleep in COPD may also be a consequence of increased end expiratory lung volume, which caused mediastinal caudal traction and transthoracic pressure changes $(81,82)$.

Whilst a clear understanding of any pathophysiological links between the two diseases remains elusive, clinical outcomes in COPD-OSA overlap are uniformly worse than in either disease in isolation, suggesting a synergistic relationship between COPD and OSA. 10-year survival in untreated COPD-OSA is significantly poorer than in COPD or OSA alone, with cardiovascular events being the most common cause of death in this cohort $(22,83)$. Causal mechanisms for increased cardiovascular risk in 
this population have not been confirmed in prospective trials however it is likely that the more severe daytime and nocturnal hypoxaemia observed in COPD-OSA overlap compared to COPD or OSA alone $(22,84)$ have three important consequences that may contribute to this risk:

\section{Chronic inflammation}

Chronic intermittent hypoxia is associated with systemic and pulmonary inflammation (85). Serum (CRP, IL-6) and airway (neutrophils, TNF $\alpha$, IL-8) inflammatory biomarkers are higher in COPD-OSA overlap than COPD, which is associated with reduced physical activity and levels correlate with duration of nocturnal hypoxia $(86,87)$. Chronic inflammation is associated with oxidative stress in both OSA and COPD which are likely linked to predispositions to vascular endothelial dysfunction and atherosclerosis observed in both conditions $(88,89)$.

\section{Pulmonary vasoconstriction}

Chronic intermittent hypoxia promotes pulmonary vasoconstriction to minimise ventilation perfusion mismatch. This increased right ventricular load leads to remodelling, resulting in higher right ventricular mass which increases in relation to severity of nocturnal desaturation (90) and may lead to myocardial fibrosis in COPD (91). These pathological changes may account for the higher prevalence of pulmonary hypertension in COPD-OSA overlap compared to OSA alone ( $42 \%$ and $13 \%$, respectively) (84) and the odds ratios of developing pulmonary hypertension in COPD-OSA overlap compared to COPD or OSA alone have been reported as 2.96 and 5.93, respectively (92). Right-heart failure is also a common consequence of hypoxaemia that occurs in both COPD and OSA and is associated with adverse clinical outcomes $(44,93,94)$.

\section{Fasting hyperglycaemia}

Nocturnal desaturation is associated with impaired glucose metabolism which manifests as fasting hyperglycaemia, with higher risk of raised fasting glucose with increasing severity of hypoxaemic events (95). Whilst impaired glucose control has established associations with adverse metabolic and cardiovascular outcomes, glucose metabolism has not been explored in the COPD-OSA population therefore any causal relation to cardiovascular outcomes remains speculative.

Whilst there have been no randomised trials evaluating the impact of continuous positive airways pressure (CPAP) therapy in COPD-OSA overlap, observational data from large cohorts indicate that 10 -year mortality is significantly higher in untreated COPD-OSA overlap compared to COPD alone, and CPAP use in COPD-OSA overlap is associated with a lower mortality risk that comparable to that for COPD alone $(22,96)$. Treatment adherence is also relevant, with poorer survival observed in those using CPAP for less than two hours a night (97). There is a propensity to develop nocturnal hypoventilation with or without daytime hypercapnia in both COPD and OSA due to loadcapacity drive imbalance, which is associated with poorer clinical outcomes as previously described. Positive airways pressure interventions (NIV and CPAP) have demonstrable benefits in COPD and OSA as single disease entities. NIV improves admission-free survival in stable hypercapnic COPD patients and those with persistent post-exacerbation hypercapnia $(59,60)$ compared to usual medical care or home oxygen. In obese patients with severe OSA and daytime hypercapnia (obesity hypoventilation syndrome), NIV improves daytime and nocturnal gas exchange and sleep quality compared to lifestyle modification alone, and there are no significant differences in risks of hospitalisation of death between OHS patients treated with CPAP or NIV (98). Given the established evidence base that positive airways pressure improves clinical outcomes in hypercapnic COPD and hypercapnic OSA, it is conceivable that it may confer such benefits in the overlap syndrome, however no randomised trials have been conducted to date. Amongst such patients previously established on home NIV, autotitrating and fixed-level NIV are associated with comparable control of daytime gas exchange, nocturnal hypoventilation and objective sleep quality, with auto-titrating devices associated with improved subjective sleep comfort and ventilator adherence (99).

Observational data demonstrate that patients with untreated COPD-OSA overlap are at increased risk of both community-treated exacerbations and those requiring hospitalisation $(22,100)$ compared to those treated with CPAP. Indeed, admission-free survival amongst overlap patients treated with CPAP is comparable to that for patients with COPD in isolation (22). In undiagnosed (and therefore untreated) OSA in COPD patients hospitalised with an exacerbation is associated with significantly higher risks of 1-, 3- and 6-month readmission compared to those without OSA. In these patients, increasing OSA severity is also associated with shorter time to readmission of death (101).

Health-related quality of life is poorer amongst COPD 
patients with co-existent OSA (23). This may be related to nocturnal hypoventilation and daytime sleepiness, and in theory should be alleviated by CPAP which improves sleep quality however prospective randomised trials evaluating patient-reported outcomes in this cohort are currently lacking.

\section{Conclusions}

COPD causes load-capacity-drive imbalance in both wakefulness and sleep, principally driven by expiratory flow limitation and hyperinflation. Sleep imposes additional burdens to the respiratory muscle pump, driven by changes in respiratory muscle tone, neural respiratory drive and consequences of the supine position. COPD patients are therefore at higher risk of decompensation during sleep and sleep disordered breathing which may manifest as altered sleep architecture, isolated nocturnal desaturation, sleep hypoventilation and restless legs. Each is associated with adverse clinical and patient-reported outcomes, including increased risk of exacerbations, hospitalisation, cardiovascular events, reduced survival and poorer quality of life. COPD-OSA overlap syndrome represents a distinct clinical diagnosis, in which clinical outcomes are significantly worse than in either disease alone, including increased mortality, risk of cardiovascular events, hospitalisation and exacerbation frequency. Sleep disordered breathing is under recognised by COPD patients and their clinicians, however early diagnosis and management, which may include non-invasive ventilation or continuous positive airway pressure, is crucial to improve patient-reported and clinical outcomes.

\section{Acknowledgments}

Funding: Dr. D'Cruz is funded by a National Institute for Health Research (NIHR) Doctoral Research Fellowship (DRF-2018-11-ST2-037).

\section{Footnote}

Provenance and Peer Review: This article was commissioned by the Guest Editor (Joerg Steier) for the series " 5 th Clinical Update Sleep" published in Fournal of Thoracic Disease. The article was sent for external peer review organized by the Guest Editor and the editorial office.

Reporting Checklist: The authors have completed the
Narrative Review Checklist. Available at http://dx.doi. org/10.21037/jtd-cus-2020-006

Conflicts of Interest: All authors have completed the ICMJE uniform disclosure form (Available at http://dx.doi. org/10.21037/jtd-cus-2020-006). The series "5th Clinical Update Sleep" was commissioned by the editorial office without any funding or sponsorship. JS served as the unpaid Guest Editor of the series and serves as an unpaid editorial board member of Fournal of Thoracic Disease. PBM reports grants and personal fees from Philips, grants and personal fees from ResMed, grants and personal fees from $\mathrm{F} \& \mathrm{P}$, grants and personal fees from B\&D Electromedical, personal fees from Santhera, grants from GSK, outside the submitted work. The authors have no other conflicts of interest to declare.

Ethical Statement: The authors are accountable for all aspects of the work in ensuring that questions related to the accuracy or integrity of any part of the work are appropriately investigated and resolved.

Open Access Statement: This is an Open Access article distributed in accordance with the Creative Commons Attribution-NonCommercial-NoDerivs 4.0 International License (CC BY-NC-ND 4.0), which permits the noncommercial replication and distribution of the article with the strict proviso that no changes or edits are made and the original work is properly cited (including links to both the formal publication through the relevant DOI and the license). See: https://creativecommons.org/licenses/by-nc-nd/4.0/.

\section{References}

1. Adeloye D, Chua S, Lee C, et al. Global and regional estimates of COPD prevalence: Systematic review and meta-analysis. J Glob Health 2015;5:020415.

2. World Health Organisation. Projections of mortality and causes of death, 2016 and 2060. Accessed 5th May 2020. Available online: http://www.who.int/healthinfo/global_ burden_disease/projections/en/

3. MacNee W. Pathogenesis of chronic obstructive pulmonary disease. Proc Am Thorac Soc 2005;2:258-66; discussion 290-1.

4. Milic-Emili J. Does mechanical injury of the peripheral airways play a role in the genesis of COPD in smokers? COPD 2004;1:85-92.

5. Jolley CJ, Moxham J. A physiological model of patient- 
reported breathlessness during daily activities in COPD. Eur Respir Rev 2009;18:66-79.

6. Global Initiative for Chronic Obstructive Lung Disease. Global Strategy for the Diagnosis, management, and Prevention of Chronic Obstructive Pulmonary Disease (2020 Report). Accessed 16th March 2020. Available online: https://goldcopd.org/gold-reports/

7. Johnson MW, Remmers JE. Accessory muscle activity during sleep in chronic obstructive pulmonary disease. J Appl Physiol Respir Environ Exerc Physiol 1984;57:1011-7.

8. Luo YM, He BT, Wu YX, et al. Neural respiratory drive and ventilation in patients with chronic obstructive pulmonary disease during sleep. Am J Respir Crit Care Med 2014;190:227-9.

9. Douglas NJ, White DP, Pickett CK, et al. Respiration during sleep in normal man. Thorax 1982;37:840-4.

10. Becker HF, Piper AJ, Flynn WE, et al. Breathing during sleep in patients with nocturnal desaturation. Am J Respir Crit Care Med 1999;159:112-8.

11. Henke KG, Dempsey JA, Kowitz JM, et al. Effects of sleep-induced increases in upper airway resistance on ventilation. J Appl Physiol (1985) 1990;69:617-24.

12. Wade OL. Movements of the thoracic cage and diaphragm in respiration. J Physiol 1954;124:193-212.

13. Stradling JR, Chadwick GA, Frew AJ. Changes in ventilation and its components in normal subjects during sleep. Thorax 1985;40:364-70.

14. Midgren B, Hansson L. Changes in transcutaneous PCO2 with sleep in normal subjects and in patients with chronic respiratory diseases. Eur J Respir Dis 1987;71:388-94.

15. Heijdra YF, Dekhuijzen PN, van Herwaarden CL, et al. Effects of body position, hyperinflation, and blood gas tensions on maximal respiratory pressures in patients with chronic obstructive pulmonary disease. Thorax 1994;49:453-8.

16. Hartman JE, Prinzen J, van Lummel RC, et al. Frequent sputum production is associated with disturbed night's rest and impaired sleep quality in patients with COPD. Sleep Breath 2015;19:1125-33.

17. Shorofsky M, Bourbeau J, Kimoff J, et al. Impaired Sleep Quality in COPD Is Associated With Exacerbations: The CanCOLD Cohort Study. Chest 2019;156:852-63.

18. Vivodtzev I, Tamisier R, Baguet JP, et al. Arterial stiffness in COPD. Chest 2014;145:861-75.

19. Connors AF, Jr., Dawson NV, Thomas C, et al. Outcomes following acute exacerbation of severe chronic obstructive lung disease. The SUPPORT investigators (Study to
Understand Prognoses and Preferences for Outcomes and Risks of Treatments). Am J Respir Crit Care Med 1996;154:959-67.

20. Lo Coco D, Mattaliano A, Lo Coco A, et al. Increased frequency of restless legs syndrome in chronic obstructive pulmonary disease patients. Sleep Med 2009;10:572-6.

21. Cavalcante AG, de Bruin PF, de Bruin VM, et al. Restless legs syndrome, sleep impairment, and fatigue in chronic obstructive pulmonary disease. Sleep Med 2012;13:842-7.

22. Marin JM, Soriano JB, Carrizo SJ, et al. Outcomes in patients with chronic obstructive pulmonary disease and obstructive sleep apnea: the overlap syndrome. Am J Respir Crit Care Med 2010;182:325-31.

23. Mermigkis C, Kopanakis A, Foldvary-Schaefer N, et al. Health-related quality of life in patients with obstructive sleep apnoea and chronic obstructive pulmonary disease (overlap syndrome). Int J Clin Pract 2007;61:207-11.

24. Price D, Small M, Milligan G, et al. Impact of nighttime symptoms in COPD: a real-world study in five European countries. Int J Chron Obstruct Pulmon Dis 2013;8:595-603.

25. Calverley PM, Brezinova V, Douglas NJ, et al. The effect of oxygenation on sleep quality in chronic bronchitis and emphysema. Am Rev Respir Dis 1982;126:206-10.

26. Cormick W, Olson LG, Hensley MJ, et al. Nocturnal hypoxaemia and quality of sleep in patients with chronic obstructive lung disease. Thorax 1986;41:846-54.

27. Valipour A, Lavie P, Lothaller H, et al. Sleep profile and symptoms of sleep disorders in patients with stable mild to moderate chronic obstructive pulmonary disease. Sleep Med 2011;12:367-72.

28. Kessler R, Partridge MR, Miravitlles M, et al. Symptom variability in patients with severe COPD: a pan-European cross-sectional study. Eur Respir J 2011;37:264-72.

29. Mulloy E, McNicholas WT. Theophylline improves gas exchange during rest, exercise, and sleep in severe chronic obstructive pulmonary disease. Am Rev Respir Dis 1993;148:1030-6.

30. Martin RJ, Bartelson BL, Smith P, et al. Effect of ipratropium bromide treatment on oxygen saturation and sleep quality in COPD. Chest 1999;115:1338-45.

31. Zhang L, Samet J, Caffo B, et al. Power spectral analysis of EEG activity during sleep in cigarette smokers. Chest 2008;133:427-32.

32. Omachi TA, Blanc PD, Claman DM, et al. Disturbed sleep among COPD patients is longitudinally associated with mortality and adverse COPD outcomes. Sleep Med 2012;13:476-83. 
33. Gries RE, Brooks LJ. Normal oxyhemoglobin saturation during sleep. How low does it go? Chest 1996;110:1489-92.

34. Aber WR, Block AJ, Hellard DW, et al. Consistency of respiratory measurements from night to night during the sleep of elderly men. Chest 1989;96:747-51.

35. Hardinge $M$, Annandale J, Bourne S, et al. British Thoracic Society guidelines for home oxygen use in adults. Thorax 2015;70 Suppl 1:11-43.

36. Lewis CA, Fergusson W, Eaton T, et al. Isolated nocturnal desaturation in COPD: prevalence and impact on quality of life and sleep. Thorax 2009;64:133-8.

37. Vos PJ, Folgering HT, van Herwaarden CL. Predictors for nocturnal hypoxaemia (mean $\mathrm{SaO} 2<90 \%$ ) in normoxic and mildly hypoxic patients with COPD. Eur Respir J $1995 ; 8: 74-7$.

38. Chaouat A, Weitzenblum E, Kessler R, et al. Sleep-related $\mathrm{O} 2$ desaturation and daytime pulmonary haemodynamics in COPD patients with mild hypoxaemia. Eur Respir J 1997;10:1730-5.

39. Fleetham J, West P, Mezon B, et al. Sleep, arousals, and oxygen desaturation in chronic obstructive pulmonary disease. The effect of oxygen therapy. Am Rev Respir Dis 1982;126:429-33.

40. McKeon JL, Murree-Allen K, Saunders NA. Supplemental oxygen and quality of sleep in patients with chronic obstructive lung disease. Thorax 1989;44:184-8.

41. McNicholas WT, Calverley PM, Lee A, et al. Long-acting inhaled anticholinergic therapy improves sleeping oxygen saturation in COPD. Eur Respir J 2004;23:825-31.

42. Goldstein RS, Ramcharan V, Bowes G, et al. Effect of supplemental nocturnal oxygen on gas exchange in patients with severe obstructive lung disease. N Engl J Med 1984;310:425-9.

43. Fletcher EC, Donner CF, Midgren B, et al. Survival in COPD patients with a daytime $\mathrm{PaO} 2$ greater than 60 $\mathrm{mm} \mathrm{Hg}$ with and without nocturnal oxyhemoglobin desaturation. Chest 1992;101:649-55.

44. Rabe KF, Hurst JR, Suissa S. Cardiovascular disease and COPD: dangerous liaisons? Eur Respir Rev 2018;27:180057.

45. Gilmartin GS, Tamisier R, Curley M, et al. Ventilatory, hemodynamic, sympathetic nervous system, and vascular reactivity changes after recurrent nocturnal sustained hypoxia in humans. Am J Physiol Heart Circ Physiol 2008;295:H778-85.

46. Tirlapur VG, Mir MA. Nocturnal hypoxemia and associated electrocardiographic changes in patients with chronic obstructive airways disease. N Engl J Med 1982;306:125-30.

47. Wouters EF. Local and systemic inflammation in chronic obstructive pulmonary disease. Proc Am Thorac Soc 2005;2:26-33.

48. Miller J, Edwards LD, Agusti A, et al. Comorbidity, systemic inflammation and outcomes in the ECLIPSE cohort. Respir Med 2013;107:1376-84.

49. Berry RB, Budhiraja R, Gottlieb DJ, et al. Rules for scoring respiratory events in sleep: update of the 2007 AASM Manual for the Scoring of Sleep and Associated Events. Deliberations of the Sleep Apnea Definitions Task Force of the American Academy of Sleep Medicine. J Clin Sleep Med 2012;8:597-619.

50. O'Donoghue FJ, Catcheside PG, Ellis EE, et al. Sleep hypoventilation in hypercapnic chronic obstructive pulmonary disease: prevalence and associated factors. Eur Respir J 2003;21:977-84.

51. Holmedahl NH, Overland B, Fondenes O, et al. Sleep hypoventilation and daytime hypercapnia in stable chronic obstructive pulmonary disease. Int J Chron Obstruct Pulmon Dis 2014;9:265-75.

52. Kitajima T, Marumo S, Shima H, et al. Clinical impact of episodic nocturnal hypercapnia and its treatment with noninvasive positive pressure ventilation in patients with stable advanced COPD. Int J Chron Obstruct Pulmon Dis 2018;13:843-53.

53. Roth T. Hypnotic use for insomnia management in chronic obstructive pulmonary disease. Sleep Med 2009;10:19-25.

54. Baillargeon J, Singh G, Kuo YF, et al. Association of Opioid and Benzodiazepine Use with Adverse Respiratory Events in Older Adults with Chronic Obstructive Pulmonary Disease. Ann Am Thorac Soc 2019;16:1245-51.

55. Ballard RD, Clover CW, Suh BY. Influence of sleep on respiratory function in emphysema. Am J Respir Crit Care Med 1995;151:945-51.

56. Hudgel DW, Martin RJ, Capehart M, et al. Contribution of hypoventilation to sleep oxygen desaturation in chronic obstructive pulmonary disease. J Appl Physiol Respir Environ Exerc Physiol 1983;55:669-77.

57. Meurice JC, Marc I, Series F. Influence of sleep on ventilatory and upper airway response to $\mathrm{CO} 2$ in normal subjects and patients with COPD. Am J Respir Crit Care Med 1995;152:1620-6.

58. Simonds AK, Elliott MW. Outcome of domiciliary nasal intermittent positive pressure ventilation in restrictive and obstructive disorders. Thorax 1995;50:604-9. 
59. Köhnlein T, Windisch W, Kohler D, et al. Noninvasive positive pressure ventilation for the treatment of severe stable chronic obstructive pulmonary disease: a prospective, multicentre, randomised, controlled clinical trial. Lancet Respir Med 2014;2:698-705.

60. Murphy PB, Rehal S, Arbane G, et al. Effect of Home Noninvasive Ventilation With Oxygen Therapy vs Oxygen Therapy Alone on Hospital Readmission or Death After an Acute COPD Exacerbation: A Randomized Clinical Trial. JAMA 2017;317:2177-86.

61. McEvoy RD, Pierce RJ, Hillman D, et al. Nocturnal noninvasive nasal ventilation in stable hypercapnic COPD: a randomised controlled trial. Thorax 2009;64:561-6.

62. Murphy PB, Brignall K, Moxham J, et al. High pressure versus high intensity noninvasive ventilation in stable hypercapnic chronic obstructive pulmonary disease: a randomized crossover trial. Int J Chron Obstruct Pulmon Dis 2012;7:811-8.

63. Adler D, Perrig S, Takahashi H, et al. Polysomnography in stable COPD under non-invasive ventilation to reduce patient-ventilator asynchrony and morning breathlessness. Sleep Breath 2012;16:1081-90.

64. Nilius G, Katamadze N, Domanski U, et al. Non-invasive ventilation with intelligent volume-assured pressure support versus pressure-controlled ventilation: effects on the respiratory event rate and sleep quality in COPD with chronic hypercapnia. Int J Chron Obstruct Pulmon Dis 2017;12:1039-45.

65. Ekkernkamp E, Storre JH, Windisch W, et al. Impact of intelligent volume-assured pressure support on sleep quality in stable hypercapnic chronic obstructive pulmonary disease patients: a randomized, crossover study. Respiration 2014;88:270-6.

66. Allen RP, Picchietti D, Hening WA, et al. Restless legs syndrome: diagnostic criteria, special considerations, and epidemiology. A report from the restless legs syndrome diagnosis and epidemiology workshop at the National Institutes of Health. Sleep Med 2003;4:101-19.

67. Malhotra A, Schwartz AR, Schneider H, et al. Research Priorities in Pathophysiology for Sleep-disordered Breathing in Patients with Chronic Obstructive Pulmonary Disease. An Official American Thoracic Society Research Statement. Am J Respir Crit Care Med 2018;197:289-99.

68. Krachman SL, Tiwari R, Vega ME, et al. Effect of Emphysema Severity on the Apnea-Hypopnea Index in Smokers with Obstructive Sleep Apnea. Ann Am Thorac Soc 2016;13:1129-35.

69. Butt M, Dwivedi G, Khair O, et al. Obstructive sleep apnea and cardiovascular disease. Int J Cardiol 2010;139:7-16.

70. Soler X, Gaio E, Powell FL, et al. High Prevalence of Obstructive Sleep Apnea in Patients with Moderate to Severe Chronic Obstructive Pulmonary Disease. Ann Am Thorac Soc 2015;12:1219-25.

71. Shawon MS, Perret JL, Senaratna CV, et al. Current evidence on prevalence and clinical outcomes of comorbid obstructive sleep apnea and chronic obstructive pulmonary disease: A systematic review. Sleep Med Rev 2017;32:58-68.

72. Redolfi S, Yumino D, Ruttanaumpawan P, et al. Relationship between overnight rostral fluid shift and Obstructive Sleep Apnea in nonobese men. Am J Respir Crit Care Med 2009;179:241-6.

73. Bernard S, LeBlanc P, Whittom F, et al. Peripheral muscle weakness in patients with chronic obstructive pulmonary disease. Am J Respir Crit Care Med 1998;158:629-34.

74. McNicholas WT. Chronic obstructive pulmonary disease and obstructive sleep apnea: overlaps in pathophysiology, systemic inflammation, and cardiovascular disease. Am J Respir Crit Care Med 2009;180:692-700.

75. He BT, Lu G, Xiao SC, et al. Coexistence of OSA may compensate for sleep related reduction in neural respiratory drive in patients with COPD. Thorax 2017;72:256-62.

76. Orr JE, Schmickl CN, Edwards BA, et al. Pathogenesis of obstructive sleep apnea in individuals with the COPD + OSA Overlap syndrome versus OSA alone. Physiol Rep 2020;8:e14371.

77. Zhu J, Zhao Z, Nie Q, et al. Effect of lung function on the apnea-hypopnea index in patients with overlap syndrome: a multicenter cross-sectional study. Sleep Breath 2020;24:1059-66.

78. Stanchina ML, Malhotra A, Fogel RB, et al. The influence of lung volume on pharyngeal mechanics, collapsibility, and genioglossus muscle activation during sleep. Sleep 2003;26:851-6.

79. Squier SB, Patil SP, Schneider H, et al. Effect of endexpiratory lung volume on upper airway collapsibility in sleeping men and women. J Appl Physiol (1985) 2010;109:977-85.

80. Biselli P, Grossman PR, Kirkness JP, et al. The effect of increased lung volume in chronic obstructive pulmonary disease on upper airway obstruction during sleep. J Appl Physiol (1985) 2015;119:266-71.

81. Van de Graaff WB. Thoracic traction on the trachea: mechanisms and magnitude. J Appl Physiol (1985) 
1991;70:1328-36.

82. Hillman DR, Walsh JH, Maddison KJ, et al. The effect of diaphragm contraction on upper airway collapsibility. J Appl Physiol (1985) 2013;115:337-45.

83. Chaouat A, Weitzenblum E, Krieger J, et al. Prognostic value of lung function and pulmonary haemodynamics in OSA patients treated with CPAP. Eur Respir J 1999;13:1091-6.

84. Chaouat A, Weitzenblum E, Krieger J, et al. Association of chronic obstructive pulmonary disease and sleep apnea syndrome. Am J Respir Crit Care Med 1995;151:82-6.

85. Eltzschig HK, Carmeliet P. Hypoxia and inflammation. $\mathrm{N}$ Engl J Med 2011;364:656-65.

86. Fitzgibbons CM, Goldstein RL, Gottlieb DJ, et al. Physical Activity in Overlap Syndrome of COPD and Obstructive Sleep Apnea: Relationship With Markers of Systemic Inflammation. J Clin Sleep Med 2019;15:973-8.

87. Wang Y, Hu K, Liu K, et al. Obstructive sleep apnea exacerbates airway inflammation in patients with chronic obstructive pulmonary disease. Sleep Med 2015;16:1123-30.

88. Schroeder EB, Welch VL, Evans GW, et al. Impaired lung function and subclinical atherosclerosis. The ARIC Study. Atherosclerosis 2005;180:367-73.

89. Drager LF, Bortolotto LA, Lorenzi MC, et al. Early signs of atherosclerosis in obstructive sleep apnea. Am J Respir Crit Care Med 2005;172:613-8.

90. Sharma B, Neilan TG, Kwong RY, et al. Evaluation of right ventricular remodeling using cardiac magnetic resonance imaging in co-existent chronic obstructive pulmonary disease and obstructive sleep apnea. COPD 2013;10:4-10.

91. Neilan TG, Bakker JP, Sharma B, et al. T1 measurements for detection of expansion of the myocardial extracellular volume in chronic obstructive pulmonary disease. Can J Cardiol 2014;30:1668-75.

92. Xu J, Wei Z, Wang X, et al. The Risk of Cardiovascular and Cerebrovascular Disease in Overlap Syndrome: A

Cite this article as: D'Cruz RF, Murphy PB, Kaltsakas G. Sleep disordered breathing and chronic obstructive pulmonary disease: a narrative review on classification, pathophysiology and clinical outcomes. J Thorac Dis 2020;12(Suppl 2):S202-S216. doi: $10.21037 /$ jtd-cus-2020-006
Meta-Analysis. J Clin Sleep Med 2020;16:1199-207.

93. Zangiabadi A, De Pasquale CG, Sajkov D. Pulmonary hypertension and right heart dysfunction in chronic lung disease. Biomed Res Int 2014;2014:739674.

94. Bradley TD, Rutherford R, Grossman RF, et al. Role of daytime hypoxemia in the pathogenesis of right heart failure in the obstructive sleep apnea syndrome. Am Rev Respir Dis 1985;131:835-9.

95. Stamatakis K, Sanders MH, Caffo B, et al. Fasting glycemia in sleep disordered breathing: lowering the threshold on oxyhemoglobin desaturation. Sleep 2008;31:1018-24.

96. Ioachimescu OC, Janocko NJ, Ciavatta MM, et al. Obstructive Lung Disease and Obstructive Sleep Apnea (OLDOSA) cohort study: 10-year assessment. J Clin Sleep Med 2020;16:267-77.

97. Stanchina ML, Welicky LM, Donat W, et al. Impact of CPAP use and age on mortality in patients with combined COPD and obstructive sleep apnea: the overlap syndrome. J Clin Sleep Med 2013;9:767-72.

98. Masa JF, Corral J, Alonso ML, et al. Efficacy of Different Treatment Alternatives for Obesity Hypoventilation Syndrome. Pickwick Study. Am J Respir Crit Care Med 2015;192:86-95.

99. Murphy PB, Arbane G, Ramsay M, et al. Safety and efficacy of auto-titrating noninvasive ventilation in COPD and obstructive sleep apnoea overlap syndrome. Eur Respir J 2015;46:548-51.

100.Donovan LM, Feemster LC, Udris EM, et al. Poor Outcomes Among Patients With Chronic Obstructive Pulmonary Disease With Higher Risk for Undiagnosed Obstructive Sleep Apnea in the LOTT Cohort. J Clin Sleep Med 2019;15:71-7.

101. Naranjo M, Willes L, Prillaman BA, et al. Undiagnosed Obstructive Sleep Apnea May Significantly Impact Outcomes in Adults Admitted for COPD in an Inner-City Hospital. Chest 2020;158:1198-207. 